\title{
$\eta$ Carinae: a very large hadron collider
}

\author{
C. Farnier ${ }^{1,2}$, R. Walter ${ }^{1,2}$, and J.-C. Leyder ${ }^{1,2,3}$ \\ ${ }^{1}$ INTEGRAL Science Data Centre, Université de Genève, Chemin d'Ecogia 16, 1290 Versoix, Switzerland \\ e-mail: Christian.Farnier; Roland.Walter@unige.ch \\ 2 Observatoire de Genève, Université de Genève, Chemin des Maillettes 51, 1290 Sauverny, Switzerland \\ 3 Institut d'Astrophysique et de Géophysique, Université de Liège, Allée du 6 Août 17, Bâtiment B5c, 4000 Liège, Belgium
}

Received 15 August 2010 / Accepted 2 November 2010

\begin{abstract}
Context. $\eta$ Carinae is the colliding wind binary with the highest mass-loss rate in our Galaxy and the only one in which hard X-ray emission has been detected.

Aims. $\eta$ Carinae is therefore a primary candidate to search for particle acceleration by probing its gamma-ray emission.

Methods. We used the first 21 months of Fermi/LAT data to extract gamma-ray $(0.2-100 \mathrm{GeV})$ images, spectra, and light-curves, then combined them with multi-wavelength observations to model the non-thermal spectral energy distribution.

Results. A bright gamma-ray source is detected at the position of $\eta$ Carinae. Its flux at a few $100 \mathrm{MeV}$ can be modelled by an extrapolation of the hard X-ray spectrum towards higher energies. The spectral energy distribution features two distinct components. The first one extends from the $\mathrm{keV}$ to $\mathrm{GeV}$ energy range, and features an exponential cutoff at $\sim 1 \mathrm{GeV}$. It can be understood as inverse Compton scattering of ultraviolet photons by electrons accelerated up to $\gamma \sim 10^{4}$ in the colliding wind region. The expected synchrotron emission is compatible with the existing upper limit to the non-thermal radio emission. The second component is a hard gamma-ray tail detected above $20 \mathrm{GeV}$. It could be explained by $\pi^{0}$-decay of accelerated hadrons interacting with the dense stellar wind. The ratio of the fluxes of the $\pi^{0}$ to inverse Compton components is roughly as predicted by simulations of colliding wind binaries. This hard gamma-ray tail can only be understood if emitted close to the wind collision region. The energy transferred to the accelerated particles ( $\sim 5 \%$ of the collision mechanical energy) is comparable to that of the thermal X-ray emission.

Conclusions. The electron spectrum responsible for the $\mathrm{keV}$ to $\mathrm{GeV}$ emission was modelled and the observed emission above $20 \mathrm{GeV}$ strongly suggests hadronic acceleration in $\eta$ Carinae. These observations are thus in good agreement with the colliding wind scenario proposed for $\eta$ Carinae.
\end{abstract}

Key words. gamma rays: stars - X-rays: binaries - X-rays: individuals: $\eta$ Carinae - X-rays: individuals: FGL J1045.0-5942 acceleration of particles

\section{Introduction}

About 30 early-type stellar systems feature synchrotron radiation in the radio domain, a signature of electron acceleration (De Becker 2007). Diffusive shock acceleration in stellar wind collisions (Benaglia \& Romero 2003), either in colliding wind binaries or OB associations, is the most likely acceleration process and a candidate for cosmic ray acceleration (Axford 1981; Casse \& Paul 1982). Gamma-rays, emitted by hadrons accelerated in stellar wind collisions, have however not yet been identified.

The gamma-ray emission expected from a colliding wind binary increases with the stellar wind mechanical luminosity, the fraction of the wind enduring collision, the photon energy density for inverse Compton emission, and the matter density for proton-proton interaction and subsequently $\pi^{0}$-decay. Located at a distance of $2.3 \mathrm{kpc}$ (Smith 2006), $\eta$ Carinae is one of the most massive (80-120 $M_{\odot}$, Davidson \& Humphreys 1997; Hillier et al. 2001) and brightest stellar system in the Galaxy and features the strongest mass-loss rate known. As, in addition, its colliding wind region is relatively wide, $\eta$ Carinae is a primary candidate for gamma-ray detection.

The Great Eruption of 1843 saw $\eta$ Carinae become the second brightest object in the sky; it was followed by another noteworthy flare in 1890 (see e.g. Davidson et al. 1999; Fernández-Lajús et al. 2010). The Homunculus is the name given to the extended bipolar nebula observed around $\eta$ Carinae: it was crafted by the colossal amount of matter $\left(10-20 M_{\odot}\right.$, Smith et al. 2003) that was ejected during the Great Eruption. The second burst led to the ejection of $\simeq 1 M_{\odot}$, creating the so-called little homunculus. $\eta$ Carinae keeps emitting matter thanks to its powerful stellar winds: the mass-loss rate is believed to be $10^{-4}$ $10^{-3} M_{\odot} \mathrm{yr}^{-1}$ (Andriesse et al. 1978; Hillier et al. 2001; Pittard \& Corcoran 2002; van Boekel et al. 2003).

Even if not all questions are settled, strong evidence suggests that $\eta$ Carinae is a binary system. For instance, radio (Duncan et al. 1995), millimetre (mm, Abraham et al. 2005), optical (Damineli 1996; Damineli et al. 2000), near-infrared (near-IR, Whitelock et al. 1994, 2004; Damineli 1996), and X-ray (Corcoran 2005) observations, obtained over the past few decades, have unveiled the existence of a period of $\sim 2022.7 \pm$ 1.3 days (Damineli et al. 2008).

The first component in the binary system is very likely a luminous blue variable (LBV, Davidson \& Humphreys 1997). The second component is probably a late-type nitrogen-rich $\mathrm{O}$ or Wolf-Rayet (WR) star (Iping et al. 2005; Verner et al. 2005). The semi-major axis of the orbit is $16.64 \mathrm{AU}$ (Hillier et al. 2001) and the eccentricity is very high $(e \sim 0.9$, Nielsen et al. 2007). Thus, the periastron distance $r_{\text {periastron }}$ is around $1.66 \mathrm{AU}$, while the primary star's radius $R_{\star, 1}$, although poorly constrained, is estimated to be in the range 0.7-1 AU (respectively, Corcoran \& Hamaguchi 2007; Damineli 1996). 
The X-ray emission of $\eta$ Carinae can be divided into two components, precisely known thanks to Chandra observations (Seward et al. 2001). The softer part $\left(k T_{\mathrm{SX}} \sim 0.5 \mathrm{keV}\right)$ prevails in the spectrum up to $1.5 \mathrm{keV}$. It is spatially extended and probably linked to the stellar winds colliding with the interstellar matter. The harder part $\left(k T_{\mathrm{HX}} \sim 4.7 \mathrm{keV}\right)$ of the spectrum dominates the $2-10 \mathrm{keV}$ domain. It is punctual, centred on the binary system, and believed to be due to the hydrodynamical shock created by the stellar winds of both components colliding with each other.

The X-ray spectrum of $\eta$ Carinae supports the colliding-wind binary scenario (Usov 1992; Stevens et al. 1992; Corcoran 2005; Pittard 2007; Parkin et al. 2009). The basic idea is that the LBV emits a rather slow and dense stellar wind $\left(v_{\infty, 1} \simeq 500 \mathrm{~km} \mathrm{~s}^{-1}\right.$, $\dot{M}_{1} \simeq 2.5 \times 10^{-4} M_{\odot} / \mathrm{yr}$, Pittard \& Corcoran 2002), which collides into the faster, shallower wind originating in the secondary component $\left(v_{\infty, 2} \simeq 3000 \mathrm{~km} \mathrm{~s}^{-1}, \dot{M}_{2} \simeq 1.0 \times 10^{-5} M_{\odot} / \mathrm{yr}\right.$, Pittard $\&$ Corcoran 2002). In this framework, the wind collision region represents the location of the hydrodynamical shock, thus of the $\mathrm{X}$-ray emitting region.

A hard X-ray tail had first been observed towards $\eta$ Carinae by BeppoSAX (Viotti et al. 2004), and subsequently unambiguously confirmed by both INTEGRAL (Leyder et al. 2008, 2010) and Suzaku (Sekiguchi et al. 2009) observations. This provides a strong evidence that the wind collision leads to non-thermal electron acceleration, and suggests the possibility of a gammaray detection.

The Carina region has been observed by several gammaray experiments. Agile detected the source 1AGL J1043-5931 $\left(\alpha=161.2, \delta=-59.7\right.$, with an uncertainty of $\left.0.4^{\circ}\right)$, which could be related to $\eta$ Carinae (Tavani et al. 2009). A two-day $\gamma$-ray flare was also observed in October 2008, although its origin is unclear. Fermi/LAT detections were reported in the Fermi bright source list (BSL, Abdo et al. 2009) and in the first year catalogue (1FGL, Abdo et al. 2010). In the BSL, the source 0FGL J1045.6-5937 lies $\approx 5.4$ arcmin from $\eta$ Carinae and is therefore not associated with it. In the 1FGL, the source 1FGL J1045.2-5942 lies 1.7 arcmin from $\eta$ Carinae, which is again too far for a formal identification. A possible association with the open cluster Trumpler 16, which may contain some energetic young pulsars, was suggested (Abdo et al. 2010). In the $\mathrm{TeV}$ domain, no detection has been reported in the vicinity of $\eta$ Carinae.

In the following sections, we describe our detailed analysis of the Fermi/LAT data, and discuss the resulting multiwavelength spectrum of $\eta$ Carinae in terms of electronic and hadronic accelerations.

\section{Fermi/LAT observations}

The Large Area Telescope (LAT) aboard the Fermi spacecraft is an electron-positron pair conversion telescope sensitive to photon energies from $20 \mathrm{MeV}$ to $>300 \mathrm{GeV}$ (Atwood et al. 2009). The LAT consists of 16 towers, each including a particle tracker and a calorimeter (allowing for the reconstruction of the direction and energy of the incident gamma-ray photons), and of an anti-coincidence shield rejecting the charged particle background.

The data are provided by the Fermi Science Support Center ${ }^{1}$, which also delivers dedicated analysis software called the Fermi ScienceTools ${ }^{2}$. The software modules allow to select events from

\footnotetext{
1 http://fermi.gfsc.nasa.gov/

2 http://fermi.gfsc.nasa.gov/ssc/data/analysis/ software
}

an incoming region of the sky, compute the corresponding livetime exposure map and the likelihood of an input sky model, and derive significance maps, source spectra, and light-curves.

\subsection{Data analysis}

We used Fermi/LAT data accumulated for the Carina region over 21 months, from August 4, 2008 to April 3, 2010. The reconstructed gamma-ray photons were selected in a circular region centred on $\eta$ Carinae with a radius of $10^{\circ}$, belonging to the diffuse class $^{3}$, and with energies between $200 \mathrm{MeV}$ and $100 \mathrm{GeV}$. This upper limit is justified by, at higher energies, the systematics of the instrument and the role of cosmic-ray events not yet being fully understood. The low energy threshold was chosen for several reasons. Firstly, $\eta$ Carinae is located in the Galactic plane, where the Galactic diffuse emission (whose spectrum can be described by a power law of photon index 2.2) is very strong. Selecting a threshold of $200 \mathrm{MeV}$ decreases the number of background events by a factor of 4 . Secondly, the Galactic diffuse emission, whose knowledge is crucial to estimating the sky model parameters at low energies, is affected by large systematic uncertainties, that influence the likelihood analysis. Thirdly, the size of the LAT point spread function decreases rapidly with increasing energy (from $100 \mathrm{MeV}$ to $200 \mathrm{MeV}$, the $68 \%$ event containment radius decreases from $5^{\circ}$ to $3^{\circ}$ ). Since the analysed region contains a large number of sources (25 sources are listed in the 1FGL within $10^{\circ}$ of $\eta$ Carinae, out of which 10 are within $3.5^{\circ}$ ), it is important to be able to distinguish the photons from different sources.

\subsection{Localisation}

The source 1FGL J1045.2-5942 ( $\alpha=161.3053, \delta=-59.7057)$ was not formally associated with $\eta$ Carinae since the latter lies slightly outside the $95 \%$ confidence region. Since we used almost twice as much data as was available when the 1FGL was generated, a more accurate source localisation can be obtained. After an initial fit of the parameters of the sources close to the location of $\eta$ Carinae, as well as of the normalisations of the diffuse Galactic and isotropic emission, we determined the position of the nearest source to $\eta$ Carinae with the tool gtfindsrc and found $\alpha=161.265$ and $\delta=-59.7015$, with a $95 \%$ confidence radius of 1.18 arcmin. $\eta$ Carinae is thus perfectly compatible with our improved position, as illustrated in Fig. 1.

Since the width of the point spread function for photons converted in the front part of the LAT is smaller than for these converted in the back, we also derived the position using the front events only, but the uncertainty did not decrease significantly. We also derived the source location for low $(<8 \mathrm{GeV})$ and high $(>10 \mathrm{GeV})$ energy events and obtained error circles also compatible with the position of $\eta$ Carinae (see Fig. 1).

The presence of the source is indisputable, with a test statistic (TS, Wilks 1938) $>2800(\approx 53 \sigma)$ for the $200 \mathrm{MeV}$ to $100 \mathrm{GeV}$ energy range. The low and high-energy components have a TS respectively of $2281(\approx 47 \sigma)$ and $73(\approx 8.5 \sigma)$. In the rest of the paper, we refer to the Fermi/LAT source as FGL $\mathrm{J} 1045.0-5942$, to reflect to its improved position.

\footnotetext{
3 http://www-glast.slac.stanford.edu/software/IS/ glast_lat_performance.htm
} 


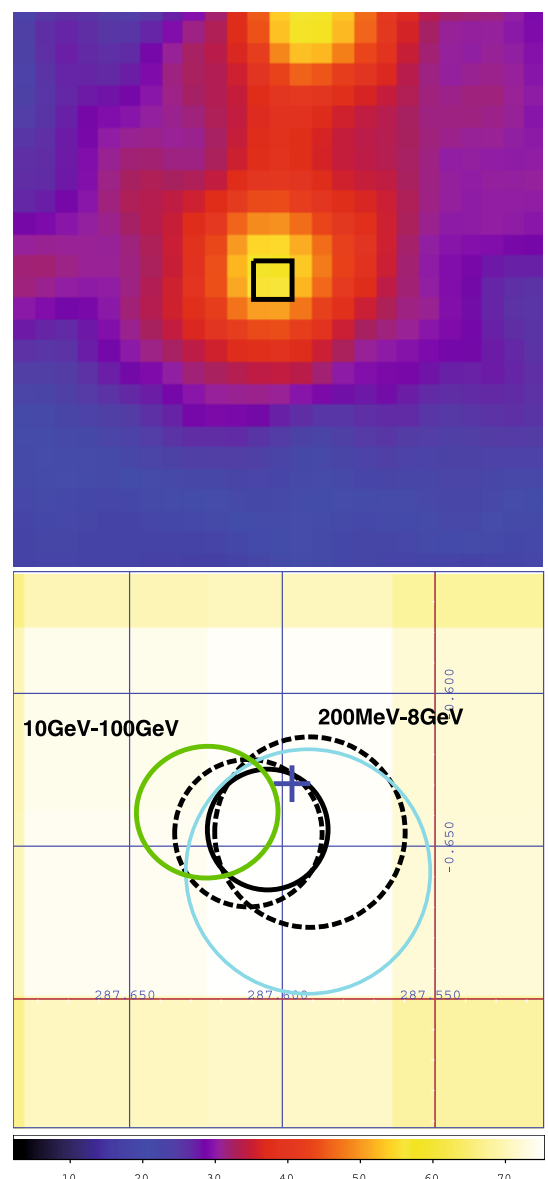

Fig. 1. Top: Fermi/LAT smoothed count map between $200 \mathrm{MeV}$ and $100 \mathrm{GeV}$ in Galactic coordinates. The bright source at the centre of the field is FGL J1045.0-5942. The source located $\sim 1.3^{\circ}$ to the North is the pulsar PSR J1048-5832. Bottom: Fermi/LAT significance map corresponding to a zoomed image of the black box shown in the upper image. The blue cross indicates the optical location of $\eta$ Carinae. The green circle is the $95 \%$ confidence region reported for 1FGL J1045.2-5942 (based on 11 months of data). The plain black circle shows the 95\% confidence region found in our analysis of the 21 month dataset. The dashed circles show the $200 \mathrm{MeV}$ to $8 \mathrm{GeV}$ and $10 \mathrm{GeV}$ to $100 \mathrm{GeV}$ confidence regions. The cyan circle corresponds to the confidence region of the hard X-ray detection obtained with INTEGRAL (Leyder et al. 2010).

\subsection{Spectral analysis}

The spectral analysis of FGL J1045.0-5942 was performed using the maximum likelihood method. The region modelling includes two types of diffuse emission (Galactic plane and isotropic) and 35 point-like sources listed in the 1FGL catalogue. While most of these sources were modelled as pure power-laws, four of them are known to be pulsars and have thus been modelled as power laws with exponential cutoffs (Abdo et al. 2010). This is particularly important for the pulsar PSR J1048-5832, located $\sim 1.3^{\circ}$ away from FGL J1045.0-5942.

Since the detection of FGL J1045.0-5942 is very significant, a spectrum could be extracted in nine spectral bins. In each bin, the TS is larger than $25(5 \sigma)$. The resulting photon spectrum and spectral energy distribution are presented in Fig. 2. Both binned (Cash 1979) and un-binned (Mattox et al. 1996) likelihoods have been used and their results are in full agreement. A curvature at low energy and a modification of the spectral slope at high energy are clearly detected.
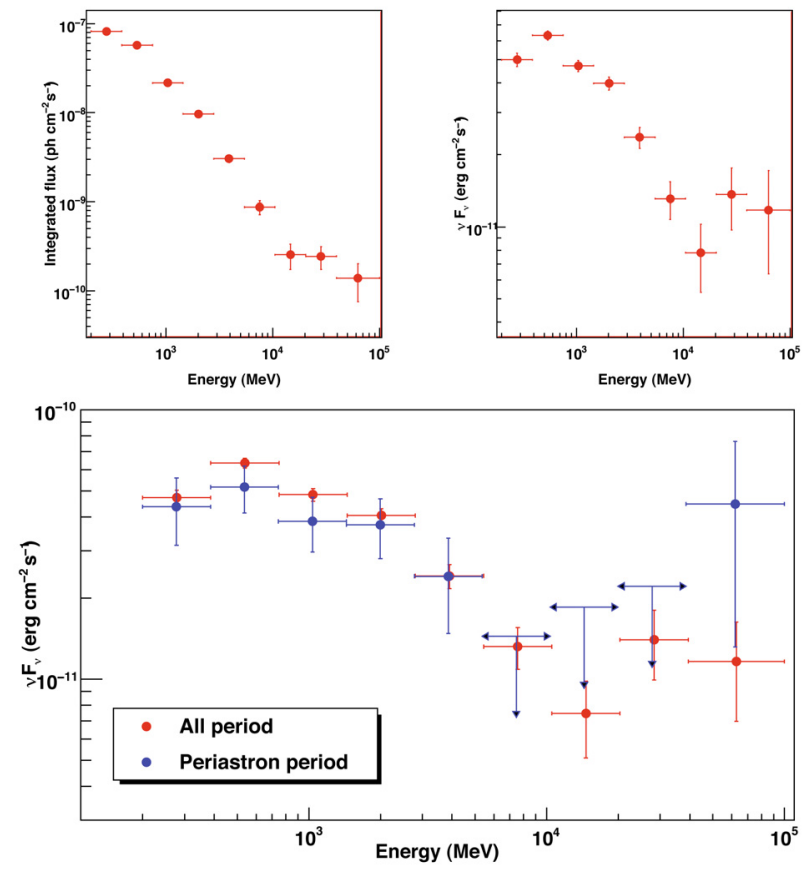

Fig. 2. Top: photon spectrum (left) and spectral energy distribution (right) of FGL J1045.0-5942 with 21 months of Fermi/LAT data. Bottom: spectral energy distribution for the complete 21 month period (red) and the periastron period (blue). Uncertainties are 95\% confidence levels.

The overall spectrum of FGL J1045.0-5942 differs from the standard power-law used to build the 1FGL catalogue, and has been modelled as a combination of two components: a power law of photon index $\Gamma$ with an exponential cutoff plus another power law at the highest energies. Since only a limited number of spectral shapes are available in the Fermi ScienceTools, the dataset has been divided into two energy ranges $(0.2-8 \mathrm{GeV}$ and $10-100 \mathrm{GeV}$ ) to determine the two spectral components independently. We maximised the sum of the likelihood in both spectral bands together so that the parameters of all sources are constrained by the complete dataset. The resulting parameters are $\Gamma=1.69 \pm 0.12, E_{\text {cut }}=1.8 \pm 0.5 \mathrm{GeV}, F_{0.2-100 \mathrm{GeV}}=$ $1.52 \times 10^{-7} \mathrm{ph} \mathrm{cm}^{-2} \mathrm{~s}^{-1}$ for the exponentially cutoff power law and $\Gamma=1.85 \pm 0.25$, and $F_{0.2-100 \mathrm{GeV}}=0.41 \times 10^{-7} \mathrm{ph} \mathrm{cm}^{-2} \mathrm{~s}^{-1}$ for the high-energy component.

\subsection{Timing analysis}

We constructed the light-curve of FGL J1045.0-5942 using the maximum likelihood technique with time bins of one month (see Fig. 3). The light-curve is consistent with a steady flux. The time-averaged flux of the source in the $0.2-100 \mathrm{GeV}$ energy range is $F_{0.2-100 \mathrm{GeV}}=(1.93 \pm 0.05) \times 10^{-7} \mathrm{ph} \mathrm{cm}^{-2} \mathrm{~s}^{-1}$.

The flux was also determined for the period when $\eta$ Carinae was at the $\mathrm{X}$-ray minimum, as measured by RXTE, (i.e. from MJD $=54843$ to MJD = 54883). The flux level obtained for this period is reported in red in Fig. 3. It is compatible with the average flux at the $95 \%$ confidence level. The spectrum detected during the periastron period is also compatible with the average spectrum obtained over a 21-month period (bottom of Fig. 2). The cutoff energy in particular did not change significantly, nor did the high-energy component, which is clearly detected above $40 \mathrm{GeV}$. 

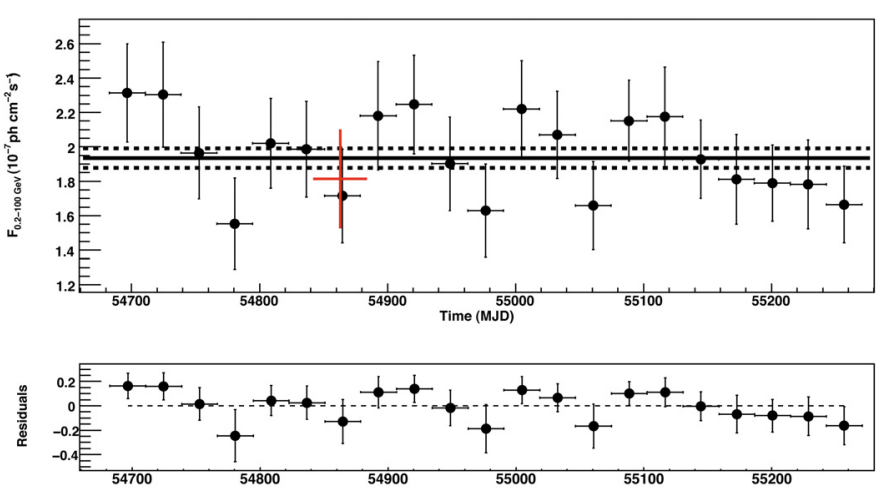

Fig. 3. Top: monthly light-curve of FGL J1045.0-5942 derived from the 21-month Fermi/LAT dataset. The red point corresponds to the flux level observed during the X-ray minimum. The black line represent the best result (with its error) obtained assuming a steady flux for the source. Uncertainties are $95 \%$ confidence levels. Bottom: residuals from the steady flux fit.

The gamma-ray emission does not vary by more than $50 \%$, much less than observed in the X-ray band at periastron, where a variability factor of larger than ten is observed.

Figure 4 shows the high-energy ( $>20 \mathrm{GeV}$ ) events superimposed on the RXTE X-ray light-curve ${ }^{4}$. Despite this high-energy component being very significant $(T S>110, \sigma>10)$, there is also no apparent correlation between the high-energy component and the orbital phase of $\eta$ Carinae.

We also searched for gamma-ray flares using a flux-aperture photometry method, but no significant deviation was found. In particular, the two-day period during which Agile reported a flare from the Carina region was investigated, but no sign of activity could be detected. During these two days, the average flux detected by Fermi/LAT was $F=(1.9 \pm 0.6) \times$ $10^{-7} \mathrm{ph} \mathrm{cm}^{-2} \mathrm{~s}^{-1}$, well below the flux detected by Agile, which is $(27.0 \pm 6.5) \times 10^{-7} \mathrm{ph} \mathrm{cm}^{-2} \mathrm{~s}^{-1}$ (Tavani et al. 2009). Since Fermi and Agile have different orbits and we used an energy threshold of $200 \mathrm{MeV}$, our analysis cannot exclude a very short and low energy flare.

\section{Discussion}

\subsection{Association with $\eta$ Carinae}

Since the spectral energy distribution observed by Fermi/LAT consists of two components (Fig. 2), a spatial coincidence of two distinct gamma-ray sources (for instance a pulsar and a blazar) cannot be excluded a priori. However, this coincidence should match the centroids of the low- and high-energy components, which are separated by less than $1.2^{\prime}$ (Fig. 1) and compatible within the uncertainties. This is rather unlikely as this would require two bright gamma-ray sources very close to $\eta$ Carinae.

Given the number of pulsars detected within $2^{\circ}$ of the Galactic plane $\left(26\right.$ or $\left.28^{5}\right)$, the probability of finding one of them in the $95 \%$ confidence region of $\eta$ Carinae is $2.5 \times 10^{-5}$. Moreover, we conducted a blind-search frequency analysis and

\footnotetext{
${ }^{4}$ http://heasarc.gsfc.nasa.gov/cgi-bin/W3Browse/ startfv.pl?file=/FTP/xte/data/archive/MissionLongData/ ETACAR.fits.gz, http://asd.gsfc.nasa.gov/Michael. Corcoran/eta_car/etacar_rxte_lightcurve/index.html

5 The 1FGL quotes two potential $\gamma$-ray millisecond pulsars within $2^{\circ}$ of the Galactic plane.
}

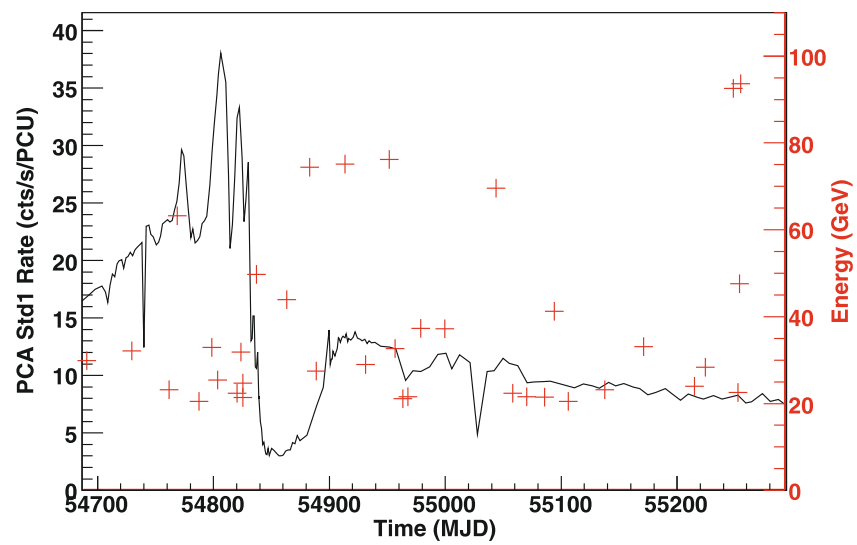

Fig. 4. The black line shows the RXTE X-ray light-curve of $\eta$ Carinae with the minimum close to the 2009 periastron passage. The red points correspond to the high-energy events $(E>20 \mathrm{GeV})$ detected by Fermi/LAT within $1^{\circ}$ of $\eta$ Carinae (note the event energy scale on the right).

did not find any evidence of a pulsed emission in FGL J1045.05942. Given the 685 sources associated with blazars in the 1FGL over the entire sky, the probability of finding a blazar at the location of $\eta$ Carinae is $2.3 \times 10^{-5}$. In addition, if the high-energy emission was related to a blazar, flaring episodes would be expected, but none is observed (see Fig. 4).

With the above probabilities and the absence of any other bright X-ray source in the INTEGRAL error circle (Leyder et al. 2010), we can safely assume that the high-energy emission detected by Fermi and INTEGRAL comes from a single source, very likely $\eta$ Carinae.

Chandra images of $\eta$ Carinae (Weis et al. 2004) reveal an extended soft shell-like component (corresponding to an external shock at the boundary of the Homunculus nebula) around the harder, point-like emission. Ohm et al. (2010) argued that the gamma-ray emission detected by Fermi could be emitted by this external shock. Their conclusion was based on the first elevenmonth spectrum, which did not exhibit the two spectral components presented above. The high-energy spectral component cannot be explained by the external shock, because the density is far too low to allow significant hadron interactions and $\pi^{0}$ cooling. In addition, Leyder et al. (2010) demonstrated that the contribution of this outer shell to the hard X-ray emission from $\eta$ Carinae was lower than $15 \%$. Thus the outer shell interpretation seems unlikely.

\subsection{Particle acceleration in $\eta$ Carinae}

We assume below that particle acceleration takes place in $\eta$ Carinae by means of diffusive shock acceleration in the colliding wind region of the binary system. In the shock region, particle acceleration is counterbalanced by four main cooling processes:

1. Inverse Compton scattering of electrons in the intense ultraviolet radiation field of the stars. The cooling time scale is

$t_{\mathrm{IC}}=\frac{3 \gamma m_{\mathrm{e}} c^{2}}{4 \sigma_{\mathrm{T}} c \gamma^{2} \beta^{2} U_{\mathrm{rad}}}=\frac{3 \pi R^{2} m_{\mathrm{e}} c^{2}}{\sigma_{\mathrm{T}} \gamma \beta^{2} L} \approx \frac{R_{10^{14} \mathrm{~cm}}^{2}}{\gamma_{10^{4}} L_{5 \times 10^{6} L_{\odot}}} \times 6 \times 10^{2} \mathrm{~s}$,

where $L$ is the stellar ultraviolet luminosity of the primary star and $R$ is its distance to the colliding wind region.

2. Proton-proton interactions and subsequent pion decay. Bednarek (2005) pointed out that high-energy hadrons in the wind of massive stars will be photo-disintegrated into 
protons and neutrons on a short timescale. The interaction timescale for protons is inversely proportional to the density of matter in the post-shock region, where the particles are trapped by the magnetic field. Hydrodynamic simulations (Pittard 2009) indicate that the density in the shock region is much higher than the unperturbed wind density, by a factor of $\delta \sim 1-100$. The $\mathrm{p}$-p cooling time scale can therefore be written as

$t_{\mathrm{pp}}=\frac{1}{\sigma_{\mathrm{pp}} \delta n c}=\frac{4 \pi R^{2} m_{\mathrm{p}} V_{\mathrm{w}}}{\sigma_{\mathrm{pp}} \delta \dot{M} c} \approx \frac{R_{10^{14} \mathrm{~cm}}^{2} V_{10^{3}} \mathrm{~km} \mathrm{~s}^{-1}}{\delta_{10} \dot{M}_{10^{-4} M_{\odot} / \mathrm{yr}}} \times 4 \times 10^{5} \mathrm{~s}$,

where $V$ is the typical wind velocity and $\dot{M}$ is the mass-loss rate of the primary star.

3. Proton diffusion away from the shock region. The diffusion timescale is related to the bulk velocity of the postshock material from the central regions towards the outside. Hydrodynamic simulations indicate that the material flows out of the shock region at a velocity smaller than, but of the order of, the pre-shock wind velocity (Pittard 2009). As the shock region has a size similar to the stellar separation, the bulk diffusion timescale can be estimated as (Bednarek 2005)

$t_{\text {bulk }}=3 R / V \approx \frac{R_{10^{14}} \mathrm{~cm}}{V_{10^{3} \mathrm{~km} \mathrm{~s}^{-1}}} \times 3 \times 10^{6} \mathrm{~s}$.

4. Electron bremsstrahlung in the post-shock region. For a density

$$
\delta n=\delta \frac{\dot{M}}{4 \pi R^{2} V m_{\mathrm{p}}} \approx \frac{\delta_{10} \dot{M}_{10^{-4}} M_{\odot} / \mathrm{yr}}{R_{10^{14} \mathrm{~cm}}} \times 3 \times 10^{9} \mathrm{~cm}^{-3},
$$

the cooling timescale is given by (Aharonian 2004) :

$$
t_{\mathrm{br}} \approx 1.2 \times 10^{15}\left(\delta n_{\mathrm{cm}^{-3}}\right)^{-1} \mathrm{~s} \approx 4 \times 10^{5} \mathrm{~s} .
$$

Particle acceleration in the shock is therefore mainly counterbalanced by inverse Compton scattering for electrons and proton-proton interactions. Equating the acceleration time $t_{\mathrm{acc}}=$ $\frac{R_{\mathrm{L}}}{c}\left(\frac{c}{V}\right)^{2}$ with $t_{\mathrm{IC}}$ and $t_{\mathrm{pp}}$ for electrons and protons respectively (where $R_{\mathrm{L}}$ is the Larmor radius) provides the maximum characteristic energy of the particle distribution.

For electrons, a power law spectrum is expected, with an exponential cutoff at an energy

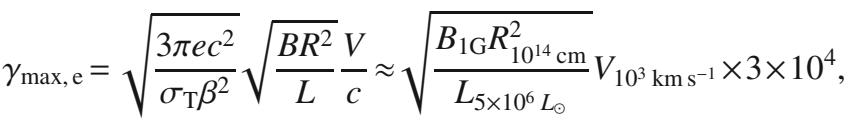

where $B$ is the magnetic field in the shock region.

The parameter $\gamma_{\max , \mathrm{e}}$ should be fairly independent of the orbital position in the dipole approximation for a magnetic field varying as $R^{-2}$. The gamma-ray spectrum will therefore exhibit an exponential cutoff at the maximal energy derived by Eichler \& Usov (1993). A cutoff energy of $\sim 1 \mathrm{GeV}$ (Sect. 2.3) corresponds to a magnetic field of $\sim 50 \mathrm{G}$ at the stellar surface.

For protons, the maximum characteristic energy is limited by proton-proton interactions to

$\gamma_{\max , \mathrm{p}}=\frac{4 \pi R^{2} e B}{\sigma_{\mathrm{pp}} \delta \dot{M}}\left(\frac{V}{c}\right)^{3} \approx \frac{R_{10^{14} \mathrm{~cm}}^{2} B_{1 \mathrm{G}} V_{10^{3} \mathrm{~km} \mathrm{~s}^{-1}}^{3}}{\delta_{10} \dot{M}_{10^{-4} M_{\odot} / \mathrm{yr}}} \times 4 \times 10^{4}$.

Although the absorption of gamma-ray photons in the stellar radiation field by means of pair production must be significant for part of the orbit, this absorption is expected to play a role

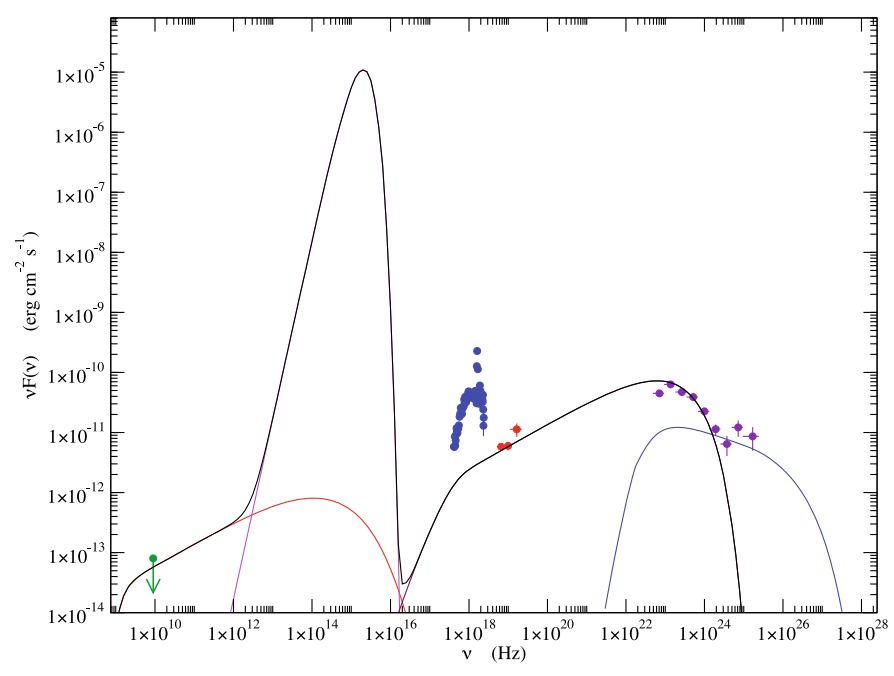

Fig. 5. Spectral energy distribution of $\eta$ Carinae including BeppoSAX/MECS (blue), INTEGRAL/ISGRI (red) and Fermi/LAT (purple) data, and a radio upper limit to the synchrotron emission (green). From low to high energies, we show the synchrotron, stellar emission, inverse Compton, and $\pi^{0}$-decay spectral components.

Table 1. Model parameters of the shock region determining the non-

\begin{tabular}{|c|c|c|c|}
\hline & Parameter & Value & \\
\hline \multirow[t]{3}{*}{ Environment } & Photon energy density & 2.7 & $\mathrm{erg} / \mathrm{cm}^{3}$ \\
\hline & Magnetic field & 0.5 & \\
\hline & Density & $3 \times 10^{9}$ & $\mathrm{~cm}^{-3}$ \\
\hline \multirow[t]{3}{*}{ Electron distribution } & Power law index & 2.25 & \\
\hline & $\gamma_{\max , \mathrm{e}}$ & $10^{4}$ & \\
\hline & Total energy & $10^{40}$ & erg \\
\hline \multirow[t]{2}{*}{ Proton distribution } & Power law index & 2.25 & \\
\hline & $\begin{array}{c}\gamma_{\max , \mathrm{p}} \\
\text { Total energy }\end{array}$ & $\begin{array}{r}10^{4} \\
1.3 \times 10^{40}\end{array}$ & $\mathrm{erg}$ \\
\hline
\end{tabular}
thermal spectral components of $\eta$ Carinae displayed in Fig. 5.

only for photons with energies $\geq 100 \mathrm{GeV}$ and does not affect the spectrum observed by Fermi. Absorption by scattering X-rays is not expected because the system remains optically thin for these photons.

We were able to reproduce the non-thermal spectral energy distribution of $\eta$ Carinae with a model consisting of two cutoff power law distributions for the electrons and the interacting protons. The model and the data are shown together in Fig. 5. The parameters of the model are listed in Table 1. The magnetic field and the electron energy distribution were adjusted to match the upper limit of the radio synchrotron emission, derived from the minimal thermal emission detected with ACTA (Duncan \& White 2003), and match the inverse Compton continuum determined by INTEGRAL and Fermi. The slope and cutoff energy of the interacting proton energy distribution were fixed to 2.25 and $10^{4}$, respectively, and its normalisation was fitted to match the high-energy gamma-ray tail using the $\pi^{0}$-decay model of Kelner et al. (2006). The proton and ultraviolet photon energy densities in the shock region were fixed to the values expected for an average distance $R=10^{14} \mathrm{~cm}$.

\subsection{Energetic considerations}

The wind momentum ratio of $\eta$ Carinae is $\eta=\left(\dot{M}_{2} V_{2}\right) /\left(\dot{M}_{1} V_{1}\right) \approx$ 0.2 (Pittard \& Corcoran 2002). The half-opening angle of the 
shock region is therefore $\sim 1 \mathrm{rad}$, the fraction of the wind involved in the wind collision region is $\sim 10 \%$, and the mechanical energy available in that region is $\sim 200 L_{\odot}$.

For a density of cold protons in the shock region similar to the one used for electrons in Sect. 3.2, the normalisation of the $\pi^{0}$-decay spectrum (Fig. 5) requires a total interacting proton energy $E_{\mathrm{p}} \sim 10^{40} \mathrm{erg}$. The energy injected in the shock to sustain the observed proton distribution is of the order of $E_{\mathrm{p}} / t_{\mathrm{pp}} \sim 10 L_{\odot}$.

The gamma-ray spectrum of $\eta$ Carinae thus indicates that $\sim 5 \%$ of the shock mechanical energy (or less than $1 \%$ of the total wind mechanical luminosity) is transferred to accelerated protons downstream. This agrees with recent numerical simulations of relativistic collisionless shocks (Spitkovsky 2008), if a significant fraction of the hadrons interacts and generates $\pi^{0}$.

Modeling the thermal X-ray emission depends on detailed hydrodynamical simulations (see e.g. Pittard \& Corcoran 2002) and could be affected by many phenomena (Parkin et al. 2009). These simulations can reproduce an X-ray luminosity of $\approx 20 L_{\odot}$ above a few keV. Our analysis hence indicates that the fraction of the shock energy accelerating protons is similar to that emitting observable X-rays in $\eta$ Carinae.

The ratio of the inverse Compton or $\pi^{0}$-decay to the X-ray emission observed in $\eta$ Carinae is larger than predicted by existing models applied to WR 140 (Pittard \& Dougherty 2006; Reimer et al. 2006). The high-energy emissivity predicted for WR140 is however not well constrained and varies by a factor of 100 depending on the model parameters. The strong inverse Compton emission and the enhanced $\pi^{0}$-emission (when compared to the X-ray emission) might be related to the strong ultraviolet photon field and the very high density in the wind collision region of $\eta$ Carinae, strengthening simultaneously the proton-proton collision rate and the absorption of the thermal emission. Detailed modelling is beyond the scope of this paper, but these observations of $\eta$ Carinae provide the first observational constraint of the fraction of the mechanical power injected into particle acceleration.

\subsection{On the detection of neutrinos}

The high-energy gamma-rays observed in $\eta$ Carinae are likely to be produced by $\pi^{0}$-decay, a process that produces as many neutrinos as gamma-rays. Thus, the detection of neutrinos would provide conclusive evidence that hadronic acceleration operates in $\eta$ Carinae.

The neutrino spectrum resulting from the decay of $\pi^{0}$ was studied by Kappes et al. (2007). In the case of $\eta$ Carinae and based on $\gamma_{\max , \mathrm{p}} \sim 10^{4}$, the expected spectrum is

$$
\frac{\mathrm{d} N_{v}}{\mathrm{~d} E_{v}} \approx\left(\frac{E_{v}}{1 \mathrm{TeV}}\right)^{-2.35} \mathrm{e}^{-\sqrt{E_{v} / 0.25 \mathrm{TeV}}} \times 10^{-12}\left(\mathrm{~cm}^{2} \mathrm{~s} \mathrm{TeV}\right)^{-1}
$$

The atmospheric neutrino background is far too high to expect any detection at such a low energy. However, since we have not measured the high-energy cutoff of the $\pi^{0}$-decay emission, we cannot exclude Cerenkov experiments being able to detect $\eta$ Carinae at energies higher $\left(\gamma_{\text {max,p }} \gtrsim 10^{5}\right)$ than anticipated. Unfortunately, even in that case, only a few neutrinos could be detected by KM3Net in 5 years of observations, not enough for a source detection.

\subsection{Other sources}

The density and magnetic field in the vicinity of the shock in $\eta$ Carinae are such that a large fraction of the energy carried by high-energy protons is converted into gamma-rays. Systems with weaker stellar winds or magnetic fields will correspond to fainter $\pi^{0}$-decay emission.

Scaling the emissivity of $\eta$ Carinae by the mass-loss rate (i.e. the wind density) suggests that LBV systems could be detected by Fermi out to the Galactic centre while, WR or OB systems could be detected within $1 \mathrm{kpc}$ and $0.1 \mathrm{kpc}$, respectively. Very few objects may therefore be detected. OB associations may have a combined stellar mass-loss rate as strong as $\eta$ Carinae and a similar efficiency in creating $\pi^{0}$ (Ozernoy et al. 1997). They could thus be detected out to distances of several kpc, although their extended emission is more difficult to measure, especially in the Galactic plane.

The mechanical energy in the stellar wind of a massive star, integrated over its lifetime, could reach some $10^{50} \mathrm{erg}$. From an energetic point of view, extrapolating the particle acceleration efficiency of $\eta$ Carinae, stellar winds ejected during the massive star evolution in a suitable environment may be as effective as a SNR to accelerate hadrons. Future Cerenkov observations will allow to determine whether stellar wind collisions could accelerate particles up to to the knee of the cosmic-ray spectrum.

\section{Conclusions}

We have detected a bright gamma-ray source at the position of $\eta$ Carinae using 21 months of Fermi/LAT data. Its flux at a few $100 \mathrm{MeV}$ corresponds very well to the extrapolation of the hard X-ray spectrum of $\eta$ Carinae (as measured by INTEGRAL and $S u z a k u$ ) to higher energies. The spectral energy distribution, which corresponds to an average of almost half of the orbit of $\eta$ Carinae, exhibits two spectral components.

The first is a power law extending from $\mathrm{keV}$ to $\mathrm{GeV}$ energies, with an exponential cutoff at $\sim 1 \mathrm{GeV}$. This component can be understood assuming inverse Compton scattering of stellar photons by electrons accelerated up to $\gamma \sim 10^{4}$ in the wind collision region. The observed cutoff energy implies a magnetic field $\sim 50 \mathrm{G}$ at the stellar surface.

The second component, a hard gamma-ray tail, is detected above $20 \mathrm{GeV}$. This bright component could be explained by $\pi^{0}$-decay of accelerated hadrons interacting with the dense stellar wind in the shock region. The ratio of the fluxes of the $\pi^{0}$ to inverse Compton components is roughly as predicted by simulations (Pittard \& Dougherty 2006; Reimer et al. 2006). Bremsstrahlung emission is expected at a much lower level and to have a cutoff energy similar to that of the inverse Compton component.

The hard gamma-ray tail can only be understood if emitted close to the wind collision region. The external shock between the Homunculus nebula and the interstellar medium indeed occurs at a density and magnetic field strength that are much too small to explain the observed emission. It is possible that a part of the soft gamma-ray emission also comes from the inverse Compton scattering of infrared photons in the external shock as suggested by Ohm et al. (2010) (see also Leyder et al. 2010). It is however difficult to account for the hard tail without a significant fraction of the soft gamma-ray emission being emitted by the colliding wind region. Detection of any hard X-ray or soft gamma-ray variability would rule out the external shock model.

The energy transmitted to the accelerated particles $(\sim 5 \%$ of the wind collision mechanical energy) is of the same order as 
that of the observed thermal X-ray emission, providing an important observational constraint for future numerical hydrodynamical models of the colliding wind region in $\eta$ Carinae.

With the exception of the short flare detected by Agile, the average hard X-ray (Leyder et al. 2010) and soft gamma-ray emission does not vary by more than $50 \%$ along the orbit, not even at periastron. This provides an interesting constraint on the evolution of both the magnetic field and the wind collision region along the orbit.

We finally suggest that Cerenkov telescopes should be able to measure the hadronic cutoff energy in $\eta$ Carinae.

\section{References}

Abdo, A. A., Ackermann, M., Ajello, M., et al. 2009, ApJS, 183, 46 Abdo, A. A., Ackermann, M., Ajello, M., et al. 2010, ApJS, 187, 460 Abraham, Z., Falceta-Gonçalves, D., Dominici, T. P., et al. 2005, A\&A, 437, 977 Aharonian, F. A. 2004, Very high energy cosmic gamma radiation: a crucial window on the extreme Universe (River Edge, NJ: World Scientific Publishing) Andriesse, C. D., Donn, B. D., \& Viotti, R. 1978, MNRAS, 185, 771 Atwood, W. B., Abdo, A. A., Ackermann, M., et al. 2009, ApJ, 697, 1071 Axford, W. I. 1981, Annals of the New York Academy of Sciences, 375, 297 Bednarek, W. 2005, MNRAS, 363, L46

Benaglia, P., \& Romero, G. E. 2003, A\&A, 399, 1121

Cash, W. 1979, ApJ, 228, 939

Casse, M., \& Paul, J. A. 1982, ApJ, 258, 860

Corcoran, M. F. 2005, AJ, 129, 2018

Corcoran, M. F., \& Hamaguchi, K. 2007, in Rev. Mex. Astron. Astrofis. Conf. Ser., 30, 29

Damineli, A. 1996, ApJ, 460, L49

Damineli, A., Kaufer, A., Wolf, B., et al. 2000, ApJ, 528, L101

Damineli, A., Hillier, D. J., Corcoran, M. F., et al. 2008, MNRAS, 384, 1649

Davidson, K., \& Humphreys, R. M. 1997, ARA\&A, 35, 1

Davidson, K., Gull, T. R., Humphreys, R. M., et al. 1999, AJ, 118, 1777

De Becker, M. 2007, A\&AR, 14, 171

Duncan, R. A., \& White, S. M. 2003, MNRAS, 338, 425
Duncan, R. A., White, S. M., Lim, J., et al. 1995, in Rev. Mex. Astron. Astrofis. Conf. Ser. 2, ed. V. Niemela, N. Morrell, \& A. Feinstein, 23

Eichler, D., \& Usov, V. 1993, ApJ, 402, 271

Fernández-Lajús, E., Fariña, C., Calderón, J. P., et al. 2010, New Astron., 15, 108

Hillier, D. J., Davidson, K., Ishibashi, K., \& Gull, T. 2001, ApJ, 553, 837

Iping, R. C., Sonneborn, G., Gull, T. R., Massa, D. L., \& Hillier, D. J. 2005, ApJ, 633, L37

Kappes, A., Hinton, J., Stegmann, C., \& Aharonian, F. A. 2007, J. Phys. Conf. Ser., 60, 243

Kelner, S. R., Aharonian, F. A., \& Bugayov, V. V. 2006, Phys. Rev. D, 74, 034018

Leyder, J., Walter, R., \& Rauw, G. 2008, A\&A, 477, L29

Leyder, J., Walter, R., \& Rauw, G. 2010, A\&A, ApJ, 718, L161

Mattox, J. R., Bertsch, D. L., Chiang, J., et al. 1996, ApJ, 461, 396

Nielsen, K. E., Corcoran, M. F., Gull, T. R., et al. 2007, ApJ, 660, 669

Ohm, S., Hinton, J. A., \& Domainko, W. 2010, ApJL, 718, L161

Ozernoy, L. M., Genzel, R., \& Usov, V. V. 1997, MNRAS, 288, 237

Parkin, E. R., Pittard, J. M., Corcoran, M. F., Hamaguchi, K., \& Stevens, I. R. 2009, MNRAS, 394, 1758

Pittard, J. M. 2007, ApJ, 660, L141

Pittard, J. M. 2009, MNRAS, 396, 1743

Pittard, J. M., \& Corcoran, M. F. 2002, A\&A, 383, 636

Pittard, J. M., \& Dougherty, S. M. 2006, MNRAS, 372, 801

Reimer, A., Pohl, M., \& Reimer, O. 2006, ApJ, 644, 1118

Sekiguchi, A., Tsujimoto, M., Kitamoto, S., et al. 2009, PASJ, 61, 629

Seward, F. D., Butt, Y. M., Karovska, M., et al. 2001, ApJ, 553, 832

Smith, N. 2006, ApJ, 644, 1151

Smith, N., Gehrz, R. D., Hinz, P. M., et al. 2003, AJ, 125, 1458

Spitkovsky, A. 2008, ApJ, 682, L5

Stevens, I. R., Blondin, J. M., \& Pollock, A. M. T. 1992, ApJ, 386, 265

Tavani, M., Sabatini, S., Pian, E., et al. 2009, ApJ, 698, L142

Usov, V. V. 1992, ApJ, 389, 635

van Boekel, R., Kervella, P., Schöller, M., et al. 2003, A\&A, 410, L37

Verner, E., Bruhweiler, F., \& Gull, T. 2005, ApJ, 624, 973

Viotti, R. F., Antonelli, L. A., Rossi, C., \& Rebecchi, S. 2004, A\&A, 420, 527

Weis, K., Corcoran, M. F., Bomans, D. J., \& Davidson, K. 2004, A\&A, 415, 595

Whitelock, P. A., Feast, M. W., Koen, C., Roberts, G., \& Carter, B. S. 1994, MNRAS, 270, 364

Whitelock, P. A., Feast, M. W., Marang, F., \& Breedt, E. 2004, MNRAS, 352, 447

Wilks, S. 1938, Ann. Math. Stat., 9, 60 